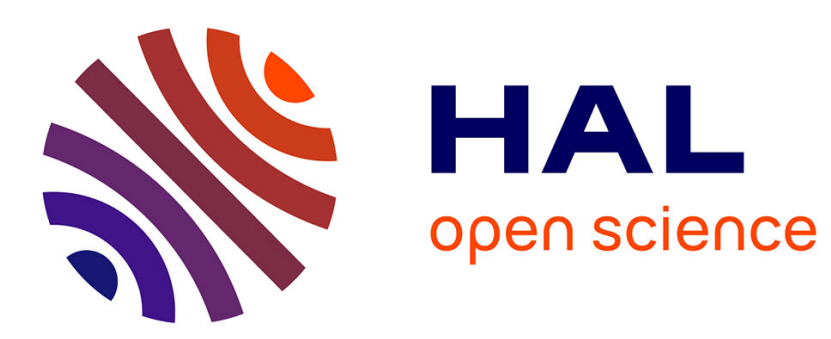

\title{
Strategy for Predictive Modeling of the Common-Mode Impedance of the Stator Coils in AC Machines
}

\author{
Nidhal Boucenna, François Costa, Sami Hlioui, Bertrand Revol
}

\section{To cite this version:}

Nidhal Boucenna, François Costa, Sami Hlioui, Bertrand Revol. Strategy for Predictive Modeling of the Common-Mode Impedance of the Stator Coils in AC Machines. IEEE Transactions on Industrial Electronics, 2016, 63 (12), pp.7360 - 7371. 10.1109/TIE.2016.2594052 . hal-01627114

\section{HAL Id: hal-01627114 https://hal.science/hal-01627114}

Submitted on 16 Mar 2018

HAL is a multi-disciplinary open access archive for the deposit and dissemination of scientific research documents, whether they are published or not. The documents may come from teaching and research institutions in France or abroad, or from public or private research centers.
L'archive ouverte pluridisciplinaire $\mathbf{H A L}$, est destinée au dépôt et à la diffusion de documents scientifiques de niveau recherche, publiés ou non, émanant des établissements d'enseignement et de recherche français ou étrangers, des laboratoires publics ou privés. 


\title{
Strategy for Predictive Modeling of the Common Mode Impedance of the Stator Coils in AC Machines
}

\author{
Nidhal Boucenna, Student Member, IEEE, François Costa, Member, IEEE, Sami Hlioui, \\ Member, IEEE, Bertrand Revol, Member, IEEE
}

\begin{abstract}
This paper presents a methodology for the predictive modeling of common mode (CM) impedance of the stator windings of $A C$ three-phase machines taking into consideration all the parasitic inductive and capacitive phenomena. An electrical model with lumped parameters (RLC) is generated, based on a physical approach of the machine, which is implantable in a SPICE type software. The prediction results are in satisfactory agreement with the measurements ones on a large frequency bandwidth [10 kHz - $100 \mathrm{MHz}$ : the lowfrequency capacitance is predicted with better than $5 \%$ accuracy, while the peaks and dips in the CM impedance plot are very well predicted beyond $5 \mathrm{MHz}$.
\end{abstract}

Index Terms-Common mode impedance, predictive modeling, windings motor modeling, lumped equivalent circuit, EMI.

\section{INTRODUCTION}

$\mathrm{N}$ OWADAYS, PWM inverters have become the standard configuration for the supply and control of AC motors. However, it has been widely recognized that the fast rise time of both voltage and current transients ( $\mathrm{dv} / \mathrm{dt}$ and $\mathrm{di} / \mathrm{dt}$ ) generated by these devices are responsible of the highfrequency electromagnetic interferences (EMI) issues [1].

In the case of three-phase $\mathrm{AC}$ induction machines, the PWM drive induces a common mode (CM) voltage at the neutral terminal of windings. This high-frequency (HF) voltage excites the different stray capacitances and HF CM currents are created flowing in metallic parts: shaft, rolling bearings, stator frame. Explanations based on physics are given for a long time in literature to understand the different phenomena involved [2]-[5]. These latter can be briefly categorized as intrinsic and extern motor EMI.

Manuscript received September 10, 2015; revised March 17, 2016 and May 24, 2016; accepted June 26, 2016. This work has been supported by the National Agency for Research, program VTT E-CEM, ref: ANR-10-VPTT-0013.

N. Boucenna was with SATIE laboratory, ENS Cachan, F-94230 Cachan, France (e-mail: nidhal.boucenna@satie.ens-cachan.fr)

F. Costa is with SATIE laboratory, ENS Cachan and Univ. Paris Est Créteil, 93000 St Denis (e-mail: francois.costa@satie.ens-cachan.fr)

S. Hlioui is with SATIE laboratory, ENS Cachan and CNAM, 75011 Paris (e-mail: sami.hlioui@satie.ens-cachan.fr)

B. Revol is with SATIE laboratory, ENS Cachan, F-94230 Cachan, France (e-mail: bertrand.revol@satie.ens-cachan.fr)
Intrinsic motor EMI include all phenomena that can cause damages inside the motor as: shaft voltage [6], [7], bearing currents and bearing voltage, which leads to bearing damages [2], [8] and electrostatic stress in windings that lead to damage their electrical insulation system [4], [5].

Extern motor EMI cover all parasitic phenomena linked to the transmission of common mode (CM) parasitic currents to the electrical environment of the motor via all parasitic capacitances toward the ground. Stator and/or rotor windings are involved but also the metallic parts and the bearings. This paper will focus on this last category of phenomena.

In order to quantify and to predict these conducted CM currents, various high-frequency models of AC motors have been presented in literature. These models can be classified into two categories: behavioral models and physics based models.

In the behavioral models, the motor is modeled by a generic arrangement of impedances identified by a set of measurements [9]-[12]. This approach requires a specific measurement set-up to extract the numerical parameters of the model out of the motors. Although these models provide a good accuracy in EMC simulation, this approach doesn't allow predicting the EMI emission at the design step of a motor or for real working conditions not identified previously.

The physics based model considers the real topology and the material properties of the motor. Each part is represented by an equivalent circuit [13]-[16]. So, this approach enables to predict all internal quantities of the motor: voltage and/or current inside windings, shaft voltage, bearing currents for any working condition. This modeling can be achieved while designing the machine and therefore takes into account the EMC issues, which is interesting to reduce the study costs in aeronautic or automotive applications. Our study is based on this last approach.

In [13] [15], the authors proposed a physical model for transient studied to predict the voltage distribution.

The physics models presented in [14] predict the value and the asymptote of the CM impedance at low frequency, in a fairly accurate manner, until the first resonance frequency, which is a proof of the accuracy of the calculation of the capacitive couplings in the motor. However, at high frequency, where the capacitive phenomena interact with the inductive phenomena, these models have an important 
discrepancy between the predicted impedance and the measured one in the high-frequency range. This discrepancy is related to the complexity and the disparity of the EM phenomena along the windings. The study presented in [16] is very interesting, however the authors have limited the study to a coil around an iron core. Regarding [17], this study is focused on bearings currents without considering the windings impedance.

In this paper, we propose a methodology for predictive modeling the common mode impedance of stator coils based on a standard AC machine motor. As it will be shown latter, this modeling strategy allows to predict the parasitic elements and to take care of the disparity of the EM phenomena along the windings.

Before introducing our strategy, we started by introducing a qualitative study, which is depicted in Section II, to clarify and to identify the different parameters that rule the CM impedance. The proposed strategy for modeling the CM impedance of the coils placed in the stator is presented in section III. This strategy is based on the use of simplified coils, which have the same electromagnetic behavior as classic motor windings. Subsequently, an electric model with lumped parameters (RLC), well-adapted to a "circuit" software (Pspice-like) has been established from the geometrical and physical parameters of these coils. The calculation of the different parameters is presented in section IV of this paper.

The proposed model allows predicting the CM impedance of the stator winding on a large high-frequency bandwidth [10 $\mathrm{kHz}-100 \mathrm{MHz}]$. The simulation results are finally compared to measurements, good agreements have been obtained.

\section{STUDIED STRUCTURE - EXPERIMENTAL MEASUREMENTS}

Predictive modeling of the high frequency behavior of windings means to model all the electromagnetic phenomena, namely capacitive phenomena and dielectric losses, inductive phenomena and iron losses in the motor environment. The capacitive phenomena are mainly governed by the dielectric proprieties of the electrical insulation system; where most of the windings in low and middle power motors are manufactured with enameled wires. The inductive phenomena are governed by the physical proprieties of the stator, rotor. Both these phenomena (capacitive and inductive) are difficult to apprehend in AC motors with industrial windings, where the conductors are randomly arranged in the slots, particularly at windings overhang (Fig. 1 (a)).

All impedance measurements presented in the paper have been achieved using an impedance analyzer (Agilent 4194A). Connection fixtures have been specifically adapted to the motor ends. Their impedances have been measured and included in the models presented hereunder.

The measured common mode impedance $\mathrm{Z}_{\mathrm{cm}}$ of an induction motor phase, at the frequency range $[1 \mathrm{kHz}-10$ $\mathrm{MHz}$, is shown in Fig. 2 (a). It can be noted that this impedance is governed by the interactions between inductive, losses and capacitive phenomena, which are illustrated by the multiple resonances and anti-resonances of the impedance modulus. The distribution of these peaks and deeps throughout the impedance spectrum reveals the disparity of the electromagnetic phenomena in the motor.

For these reasons, in order to explain the complexity of these phenomena, we have chosen to carry out a progressive approach in which we can control the geometrical parameters (turns number, arrangement, etc.) and some physical parameters (material properties).Thus, we choose to focus our study on a simplified winding made with rigid wire insulated by Polyvinyl Chloride (PVC) (Fig. 1 (b)). This winding is composed of seven coils connected in series. It has been wounded directly inside the stator of the induction motor as shown in Fig.1 (b). This wire has allowed us to realize identical coils and to have a fixed and known arrangement of the turns in each coil. Due to the thickness of the wire insulation, which is $0.72 \mathrm{~mm}$ of PVC, each coil is made by four turns. Moreover, the conductors section has been chosen in order to fill all the available cross-section of the slots as shown in Fig.1 (c). Thus, it doesn't need to insert any insulation between turns and slots.

The CM impedance $Z_{\mathrm{cm}}$ of this winding is reported in Fig. 2 (b) on the frequency rage $[10 \mathrm{kHz}$ to $100 \mathrm{MHz}]$. We can observe that this impedance is similarly governed by the interactions between the capacitive and the inductive phenomena. The evolutions of this curve are quite-identical to the ones of the CM impedance of the real motor phase presented in Fig. 2 (a) but shifted to the high frequencies (roughly 2 decades). This means that the electromagnetic phenomena in this simplified winding are equivalent to those of the industrial winding. Indeed, we can see that both impedances have firstly a capacitive behavior followed by successive resonances at different frequencies. Thus, we can use this simplified winding and build our methodology around it.

Otherwise, because all the coils are identical, we chose to reduce the studied model to one coil. The geometric parameters of one coil are reported in Table I.

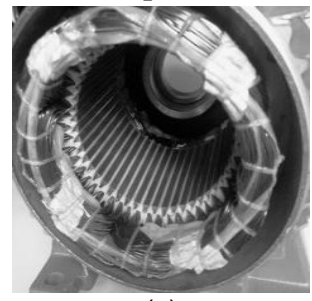

(a)

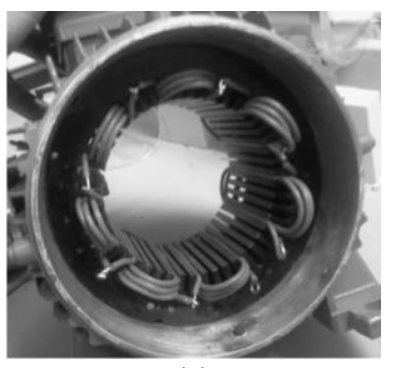

(b)

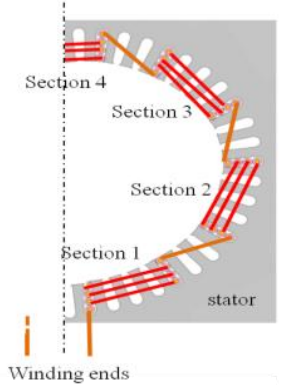

(c)
Fig. 1. (a) induction motor $1,1 \mathrm{~kW}$, (b) simplified winding composed of 7 experimental coils, (c) wire disposition of each coil in the stator (half cross section) 

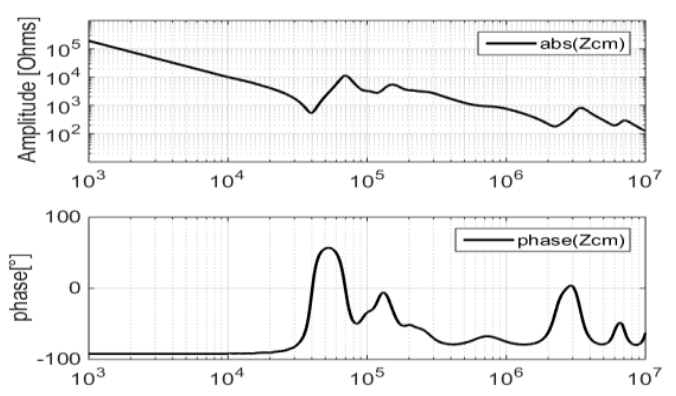

(a)

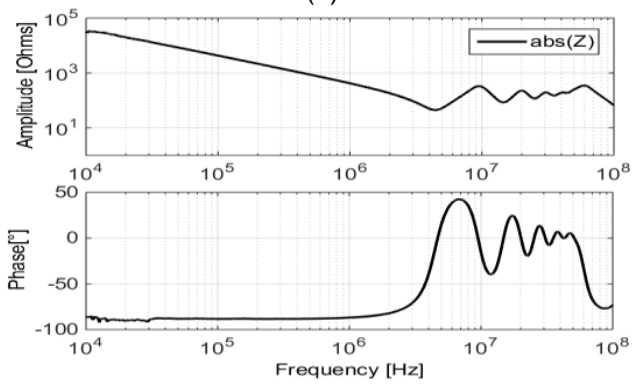

(b)

Fig. 2. (a) CM impedance of one phase-modulus \& phase, (b) CM impedance of the simplified winding-modulus \& phase

TABLE I

GEOMETRIC PARAMETERS OF THE EXPERIMENTAL COIL (4 TURNS)

\begin{tabular}{ll}
\hline Wire diameter & $1.33 \mathrm{~mm}$ \\
Wire diameter with the insulator & $2.77 \mathrm{~mm}$ \\
Conductor length in the slots & $70 \mathrm{~mm}$ \\
Length of each winding overhang & $65 \mathrm{~mm}$ \\
\hline
\end{tabular}

\section{A. Initial simplified approach}

In order to investigate the basic configuration, we realized a quantitative study to identify the critical parameters and to understand the repartition of electromagnetic phenomena in each coil. The upper limit in the frequency analysis has been limited to $100 \mathrm{MHz}$ for all the study presented herein. This value has been chosen in order to anticipate the conducted emissions of wide band-gap semiconductors ( $\mathrm{SiC}, \mathrm{GaN})$.

Firstly, we measured the CM impedance of this basic coil, which is carried out between one coil terminal and the frame of the motor. As we can note in Fig. 3, there are two critical points: a resonance at $33.8 \mathrm{MHz}$ and an anti-resonance at $66 \mathrm{MHz}$.

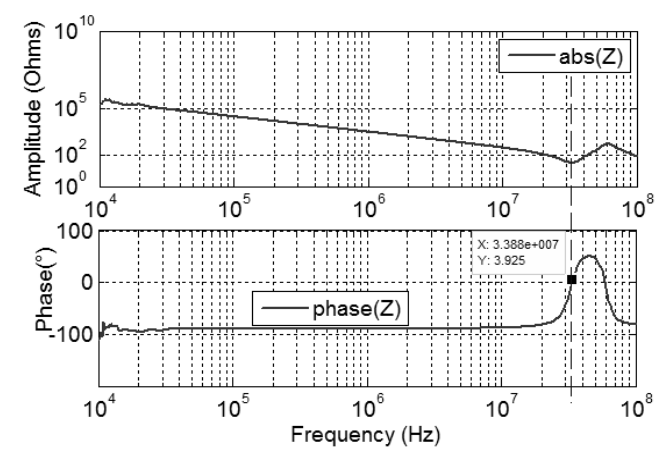

Fig. 3. CM impedance of the experimental coil

For frequencies lower than $10 \mathrm{MHz}$ (Fig.3), the coil has a capacitive behavior. It is mainly due to the parasitic capacitances between the conductors and the slot's walls. However, for frequencies higher than $10 \mathrm{MHz}$, the inductive effects become dominant in the impedance and interact with the capacitive phenomena

Further experiments have shown that the contribution of the overhanging regions of the winding is more significant than the inductive phenomena in the stator within this frequency band. This suggests that at high frequency, the leakage magnetic energy is located in air inside slots and in overhanging regions of windings and not in metallic parts. These experiments also highlighted that the inductive phenomena at high frequency are different from the ones at low frequency involving mainly the iron regions of the machine.

For this purpose, in the next step of modeling, we will consider the distribution of the HF magnetic phenomena in slots and in overhanging regions separately.

\section{MODELING STRATEGY}

To take into account the electromagnetic phenomena diversity in the coil, we divided the experimental coil in two regions (Fig. 4);

- Iron region: The capacitive couplings are dominant in these regions due to the proximity of conductors with the slot's walls. Thus, the CM capacitive currents are predominant in these regions. Inversely, the $\mathrm{HF}$ magnetic effects are small because the leakage magnetic field is confined inside the slots due to the shielding effect of the metallic slot's walls.

- Air region: The capacitive couplings are significantly smaller in these regions because the conductors are outside the metallic parts of the stator. In these regions, the conduction currents and the inductive phenomena are predominant.

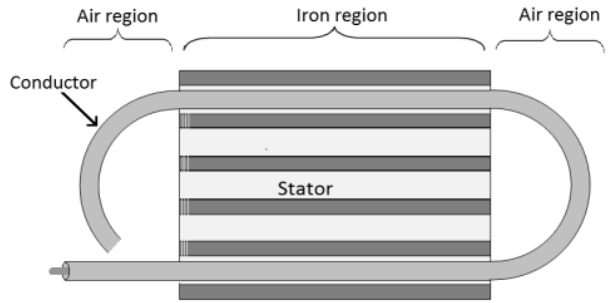

Fig. 4. Description of a turn partition: radial view of a section, see Fig. 1 (c)

The modeling strategy proposed herein is to model each region by a lumped parameters circuit. This approach is valid as long as the portion length of the conductor, which has to be modeled, is lower than the wavelength of the maximum studied frequency of electrical quantities. In our case, the measurement is carried out until $100 \mathrm{MHz}$. Considering that the celerity of the voltage wave propagation equal to $2 * 10^{8}$ $\mathrm{m} / \mathrm{s}$, the wavelength is equal to $2 \mathrm{~m}$. This length is roughly 30 times greater than the conductor length in each region (see Table I). Consequently, we can assign to each region an RLC circuit where each lumped parameter has a physical meaning. Thus, we consider the following parts: 


\section{A. Iron region (conductors in the slots):}

Each conductor in the slot is modeled by a cell that contains the following elements:

- Conductor resistance $R_{s e}$

- The inductance $L_{s e}$ of the circuit loop formed by the conductor and the slot walls (see Fig. 5). This point is detailed in the next paragraph (identification of inductive phenomena).

- The capacitance $C_{p e}$ that represents the capacitive coupling between the conductor and the slot walls. The resistor $R_{p e}$ is paralleled with capacitance $C_{p e}$ to model the dielectric losses.

At last, there is one conductor "Forward" and one conductor "Return" for each coil turn, the iron region of each turn is model by two RLC cells. Thus, the lumped parameters of the conductor "Return" are noted $R_{s e}$ ', $L_{s e}$, $C_{p e}$ '. In our experiment, the impedance measurement has been achieved with low-level signals (Impedance analyzer). Since the magnitude of the CM impedance is very high (see Fig. 3), the current's magnitudes, and therefore the magnetic field, remain low. For this purpose, we hypothesized that the iron losses remain relatively low and we choose to neglect them in the model.

\section{B. Air region (coil's overhangs ):}

Each conductor in the "Air region" is modeled by a cell that contains the following elements;

- Conductor resistance $R_{s t}$

- The inductance $L_{s t}$ of the half-loop formed by the conductor in this region.

Consequently, the CM electrical model of one complete turn consists of the series arrangement of the electrical circuits of each region as presented in Fig. 5.
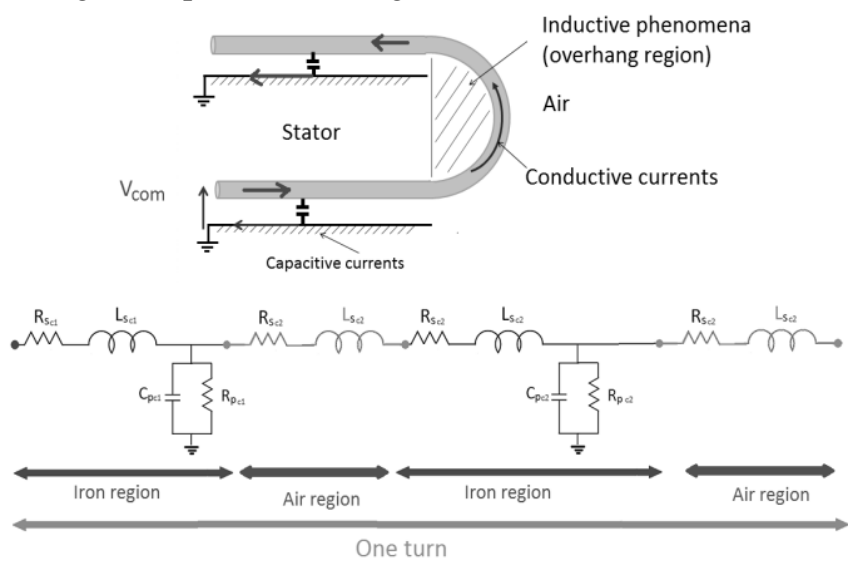

Fig. 5. Electrical model of one turn in the stator

In addition to these parameters, the inter-turns couplings are taken into account by means of the following parameters (Fig. 6).

- Inter-turns capacitive couplings:

It is modeled by the capacitance $C_{t e}$ for adjacent conductors in the iron regions and by the capacitance $C_{t t}$ for adjacent conductors in the air regions (end windings). These capacitances are paralleled with the resistances $R_{t e}$ and $R_{t t}$ respectively, whose are corresponding to the dielectric losses.

- Inter-turns inductive couplings:

Coupling coefficients have been used to quantify the inductive couplings between the relevant inductances in each region.

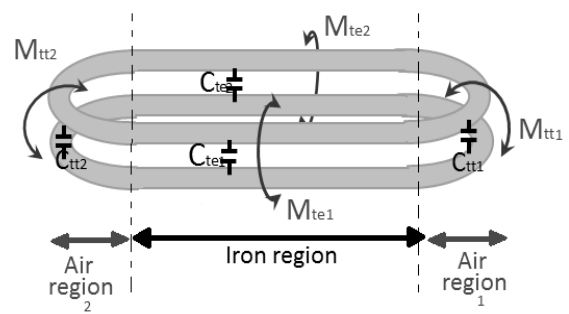

Fig. 6. Capacitive and inductive coupling between two turns

The different steps that have to be followed to obtain the common mode circuit are represented in the diagram Fig.7

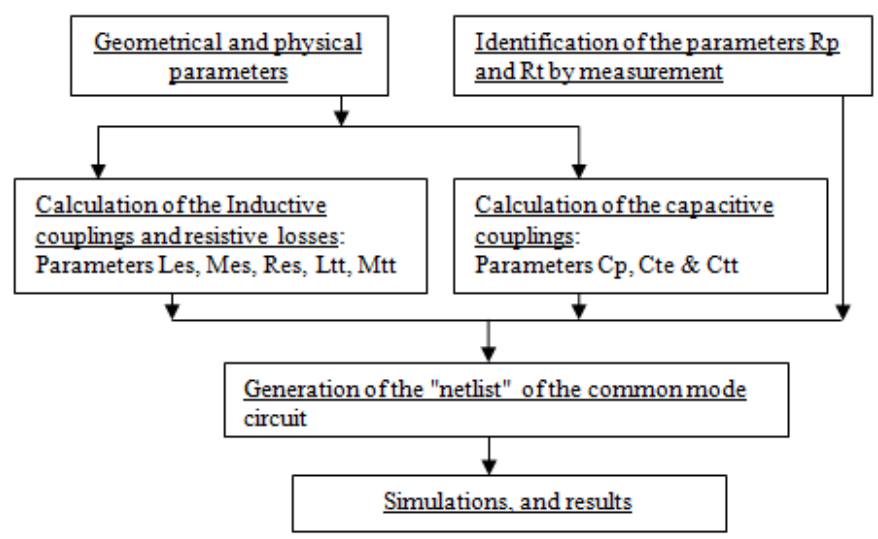

Fig. 7. Steps used to generate the "netlist" file

Despite the simplicity of the cell model and the low number of turns (4) of the experimental coil, the number of electrical parameters of the electrical model of one coil is important (64 parameters besides inductive couplings) (Fig.8). Therefore, it is very difficult to use the graphic interface of a circuit simulator for the analysis of this model.

In our study, we used a freeware SPICE simulator (simulation program with integrated circuits emphasis). In addition to the graphic interface, this software allows analyzing "netlist" files, wherein all the electrical parameters of the circuit model are written according to the classical protocol.

The "netlist" file representing the complete coil is generated using a MATLAB program. This latter carried out an analysis of the input data then creates arrangements matrix and assigns the value and node's connections of each electrical element of the model. This program enables us to treat any values of the turn number. 


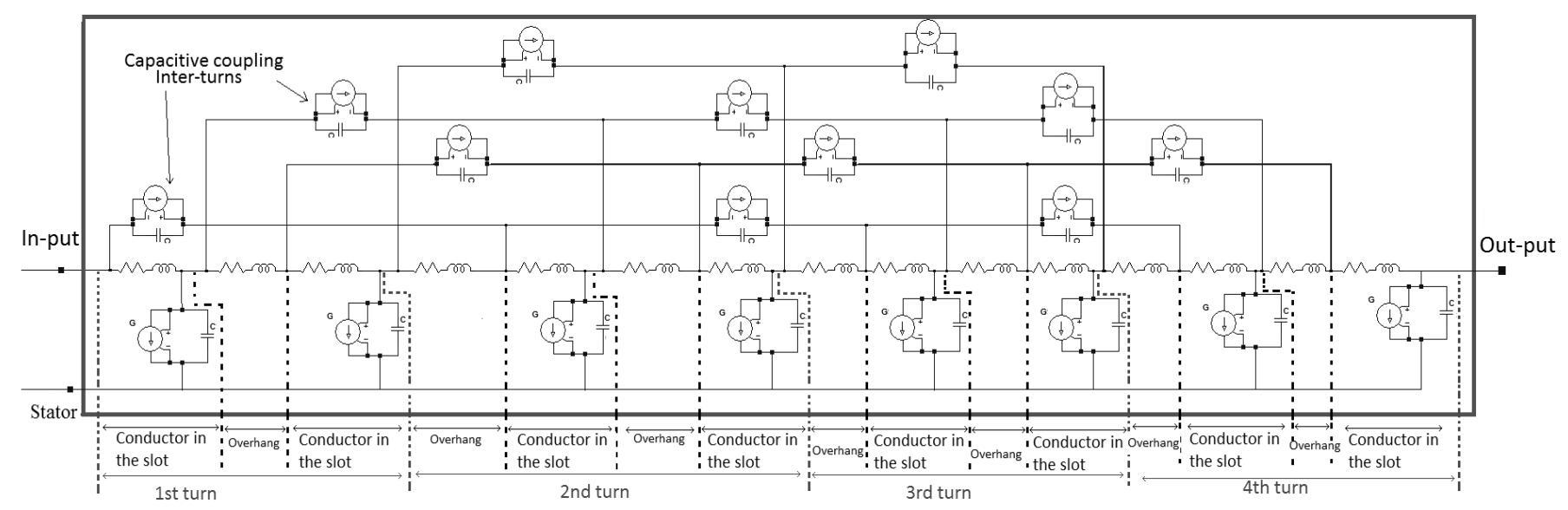

Fig. 8. Electrical circuit of the 4 turns coil

Now that the architecture of the CM model has been set we present in the next section the successive steps to calculate its different parameters from the geometrical and physical proprieties of the materials.

\section{NUMERICAL AND ANALYTICAL CALCULATIONS OF THE CIRCUIT PARAMETERS}

Because the CM impedance of the coil has a capacitive behavior until few $\mathrm{MHz}$, we started by calculating the capacitive parameters then the inductive parameters of the model.

\section{A. Capacitive couplings:}

The capacitive couplings are governed by the dielectric properties of the insulation system. In our case, this latter is composed of the conductor insulation (PVC in our experiments) and air.

\section{1) Calculation of inter-conductor capacitive couplings $C_{t}$ and dielectric losses $R_{t}$}

These two parameters $\left(C_{t}\right.$ and $\left.R_{t}\right)$ are corresponding to the impedance between two adjacent conductors at different potential.

The capacitance parameters, which are denoted $C_{t e}$ in the iron region and $C_{t t}$ in the air region, have been calculated by finite element (FE) method with the FEMM software [18]. The 2D electrostatic model reported in Fig. 9 was used for calculating both parameters. In this study, we used a constant value $\left(\varepsilon_{r}=3.4\right)$ as the relative permittivity of PVC, which was determined experimentally.

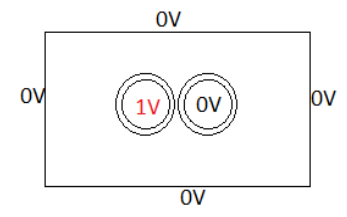

Fig. 9. Outline of the 2D test model Electrostatic

The simulation conditions used in the calculation of each parameter are reported in Table II. In addition of the length difference of the conductor in the two regions, the spacing between conductors in the air region was supposed to be greater than the spacing in the iron region where they are compressed and maintained by the slot walls (see in Fig 1(b,c) and Fig 4).

The capacitance $\mathrm{C}$ is extracted from the relation between the electric charge $\mathrm{Q}$ and the potential of the conductor $\mathrm{V}$ $(\mathrm{C}=\mathrm{Q} / \mathrm{V})$.

TABLE II

\begin{tabular}{|c|c|c|c|c|}
\hline \multirow{3}{*}{ parameters } & \multicolumn{2}{|c|}{ The simulation conditions } & \multirow{2}{*}{\multicolumn{2}{|c|}{$\begin{array}{l}\text { Capacitance } \\
\text { value }[\mathrm{pF}]\end{array}$}} \\
\hline & \multirow{2}{*}{$\begin{array}{l}\text { Length of the } \\
\text { conductors in each } \\
\text { region }[\mathrm{mm}]\end{array}$} & \multirow{2}{*}{$\begin{array}{l}\text { Spacing between } \\
\text { the conductors } \\
{[\mathrm{mm}]}\end{array}$} & & \\
\hline & & & FE & $\begin{array}{l}\text { Measu- } \\
\text { rement }\end{array}$ \\
\hline $\mathrm{C}_{\mathrm{te}}$ & 70 & 0.1 & 3.0 & 2.9 \\
\hline $\mathrm{C}_{\mathrm{tt}}$ & 65 & 0.2 & 2.6 & 2.3 \\
\hline
\end{tabular}

As we can note in the Table II, the calculation results of the capacitances $C_{t e}$ and $C_{t t}$ are in good agreement with the measurements. Thus, in the following sections, we have retained the numerical calculation of these parameters, which are considered constant over the entire frequency range.

The dielectric losse parameter, which is denoted by $R_{t e}$ in the iron region and $R_{t t}$ in the air region, has been extracted from the impedance measurement between two conductors. Indeed, the impedance analyzer (Agilent 4194A) allows indentifying the parameters of a behavioral model of the measured impedance, which is composed of the capacitance $C_{\text {parallel }}$ in parallel with the resistance $R_{\text {parrallel }}\left(\mathrm{C}_{\text {parallel }} / / R_{\text {parallel }}\right)$. Thus, the value of $R_{\text {parallel }}$ is representative of the dielectric losses between the two conductors (PVC + air). The measurement result for each region is reported in Fig. 10.

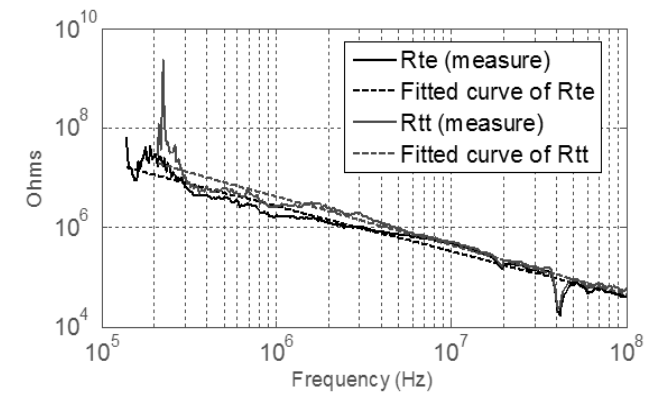

Fig. 10. Measured vs. trend curve of parameters $R_{t e}$ et $R_{t t}$

As we can note, these parameters are nonlinear and strongly depending on frequency. Also, we can note that the 
measurements contain some noise for frequencies under 300 $\mathrm{kHz}$. This is due to the value of the measured impedance which is very close to the limit of the impedance analyzer.

To use these experimental data in a circuit simulation, we have identified numerically functions (1) to fit each curve as we can see in Fig. 10. These frequency domain functions allow transcribing averagely the frequency dependence of the dielectric losses.

$$
\left\{\begin{array}{l}
R_{t e}(f)=3.531 * 10^{12} * f^{-0.98} \\
R_{t t}(f)=7.5616^{*} 10^{11} * f^{-0.9}
\end{array}\right.
$$

Where, " $f$ " is the frequency.

These functions decrease almost by $1 / \mathrm{f}$ (coefficient -0.98 and -0.9 ), and thus, the dielectric losses are quite-proportional to the frequency. The coefficients of the equation (1) are depending of the dielectric properties and the dimension of the insulation system of the conductors and their disposition.

Instead of using a simple resistance, which cannot be frequency-dependant, a voltage dependent current-source element (Fig. 11 (b)) from the LTSPICE library was used to take into account the frequency dependence of the parameter $R_{t e}$ and $R_{t t}$ (Fig. 11 (a)). The obtained model, which is composed of the capacitance element $C_{t}$ and the voltage dependent current-source, Fig. 11 (b), allows taking into account the electrostatic phenomena between the two conductors and the frequency dependent dielectric losses.

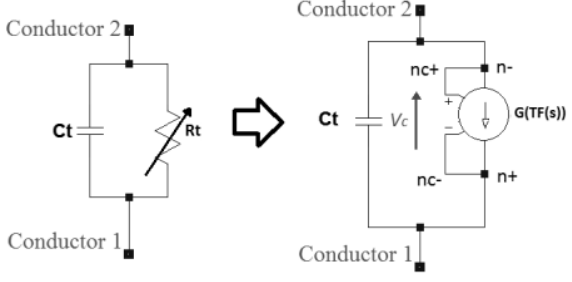

(a)

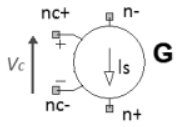

(b)
Fig. 11. (a) "voltage dependent current source" from the LTSPICE library used for modeling the parameters $R_{t e}$ and $R_{t t}$ (b) Full model of non-linear capacitance $\left(\mathrm{C}_{\mathrm{t}} / / \mathrm{R}_{\mathrm{t}}\right)$

This circuit element (Fig. 11 (b)) asserts an output current $I_{s}$ between the nodes $(n+)$ and $(n-)$ that depends on the input voltage between nodes (nc+) and (nc-), which is the voltage across the capacitance $C_{t}$, and a transfer function $\mathrm{TF}(\mathrm{s})$ which has to be defined beforehand. The value of the current $I_{s}$ is given by (2):

$$
I_{s}=\frac{1}{T F(s)} V_{s}
$$

In the case of the functions expressed by (1), the transfer functions are obtained by doing a substitution of the frequency variable (f) by the Laplace variable (s) reported in (3).

$$
f=\frac{s}{j 2 \pi}
$$

Therefore, we found

$$
\left\{\begin{array}{l}
R_{t e}(f)=T F_{t e}(s)=3.531 * 10^{12} *\left(\frac{s}{j 2 \pi}\right)^{-0.98} \\
R_{t t}(f)=T F_{t t}(s)=7.5616 * 10^{11} *\left(\frac{s}{j 2 \pi}\right)^{-0.9}
\end{array}\right.
$$

\section{2) Calculation of capacitive couplings $C_{p}$ and dielectric losses $R_{p}$ between conductors and the slot's walls (iron region)}

The parameters $C_{p}$ are also calculated by FE method and compared to measurements. The results are reported in Table III (Where $C_{p l}$ is the capacitance between the conductor, in the bottom of slot, and the slot wall). As we can see, the numerical results are in good agreement with the experimental results. The slight difference between measured and numerical values of the capacitances $C_{p 2}$ and $C_{p 3}$ is due the geometrical position of the conductors in the slot that is not faithfully modeled. Note that the position of the conductors is supposed to be the same in each slot ("Forward"/"Return"). Thus, the

\begin{tabular}{|c|c|c|c|c|c|}
\hline \multirow{2}{*}{ Capacitance } & \multicolumn{2}{|c|}{ Value $[\mathrm{pF}]$} & \multirow{2}{*}{ Capacitance } & \multicolumn{2}{|c|}{ Value $[\mathrm{pF}]$} \\
\hline & Measure & FE & & Measure & FE \\
\hline $\mathrm{C}_{\mathrm{p} 1}$ & 6.9 & 6.4 & $\mathrm{C}_{\mathrm{p} 3}$ & 4.4 & 5.7 \\
\hline $\mathrm{C}_{\mathrm{p} 2}$ & 5.8 & 4.5 & $\mathrm{C}_{\mathrm{p} 4}$ & 8.7 & 8.9 \\
\hline
\end{tabular}
capacitances $C_{p}$ ' are similar to the capacitance $C_{p}$.

TABLE III

MEASURED VS. FE CALCULATION OF THE CAPACITANCE $\mathrm{C}_{\mathrm{P}}$

As previously, a loss parameter $R_{p}$ is associated in parallel to $C_{p}$. It combines the dielectric loss in the insulation layer between the conductor and the slot wall (capacitor $C_{p}$ ) and the metallic loss in the slot wall due to the displacement current through $C_{p}$. This value is difficult to extract for each conductor because it depends on the conductor position in the slot. For simplicity reason, we have considered that this parameter is the same for each conductor placed near the slot's walls, and equal to the reference value of the parameter $R_{p l}$, which is corresponding to the dielectric losses extracted from the measured impedance (Fig. 12), between a conductor placed in the bottom of the slots and the stator.

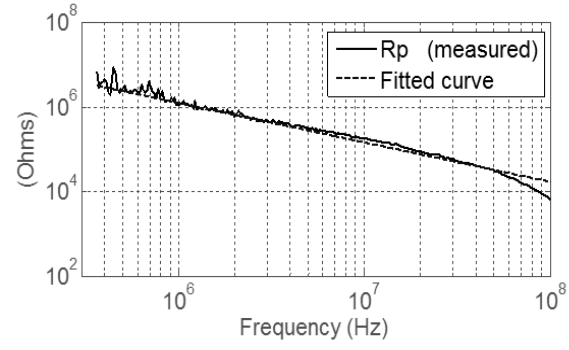

Fig. 12. Measured vs. trend curve of the parameter $R_{p}$

A numerical function (5) was also used hereunder to fit the experimental curve of the parameter $R_{p}$ (Fig. 12).

$$
R_{p}(f)=T F_{p}(s)=4.643 \times 10^{11}\left(\frac{s}{j 2 \pi}\right)^{-0.93}
$$

\section{$B$. Inductive couplings:}

The inductance of a current loop $(I)$ is given by the ratio of the total flux, embraced by the area enclosed by this loop, and 
this current. Based on this definition, the current loops have to be identified correctly. The CM voltage is applied between the input terminal of the coil and the frame. Thus, in the iron region, capacitive currents flow through the stray capacitances $\left(C_{p}\right)$ to the stator to reach the ground. The circulating of these currents forms a current loop in each slot.

Because the output terminal of the coil is at a floating potential (it is not connected), the conduction currents are very low in the coil; they are distributed all along the coil. However, in the air region where the capacitive couplings between the conductors and the stator are low, the conduction currents are the main origin of the inductive phenomena in the overhang region of the coil.

From this analysis, we can distinguish two kinds of current loops (Fig. 13):

- Current loops bounded by the conductor in iron region, and the slot's walls. The inductance of this current loop is notated $L_{e s}$ in the slot "Forward" and $L_{e s}{ }^{\prime}$ in the slot "Return".

- Current loops formed by the overhang region. The inductance of this current loop is notated $L_{t t}$

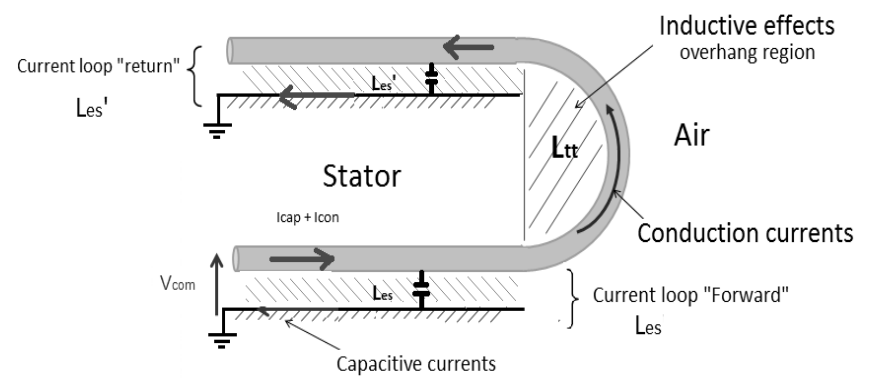

Fig. 13. Descriptive diagram of different current loops in one turn placed in the stator

\section{1) Calculation of the inductive parameters in the "Iron} region"

Inductance $\underline{L}_{\underline{e s}}$ and $\underline{L}_{\underline{e s}}{ }_{-}:$

We only used a numerical approach in the identification of the inductance parameters $L_{e s}$ and $L_{e s}$ '. A 2D magnetodynamic model was developed using once again the software FEMM. This model is based on the geometrical and physical parameters of the frame, the stator and the experimental coil, see in Fig. 14.

In order to get directly values corresponding to the $3 \mathrm{D}$ case and thanks to the rotation symmetries, the depth of the 2D meshed model is defined equal to the effective length of the stator multiplied by the stacking factor (98\%). Then, the magnetic energy stored between iron sheets is neglected.

In order to calculate the inductance parameter $L_{e s l}$ of the current loops formed by the propagation path of the capacitive currents between the first conductor of the "Forward" slot and the slot's wall, we have carried out as following. This first conductor is set to $1 \mathrm{~A}$ current $(+\mathrm{I}=1 \mathrm{~A})$ where all the other conductors are set to zero current, even the conductor in the "Return" slot. The stator is set to $-1 \mathrm{~A}$ current $(-\mathrm{I}=-1 \mathrm{~A})$. Thus, we have a current $(+\mathrm{I})$ that flows through the conductor and loops through the stator (see Fig. 14). After the resolution, the solver calculates the total magnetic energy. Then, the inductance $\mathrm{L}_{\mathrm{es} 1}$ is calculated using (6).

$$
L_{e s n}=2 W_{\text {mag }} /\left(I_{n}\right)^{2}
$$

This methodology is used to calculate all the other inductances $L_{e s}$ corresponding to the other current loops (formed by each conductor). The calculation results are presented in Table IV. The simulation is time consuming mainly at high frequencies where the number of elements is very important because skin and proximity effects have been considered in the meshing. These calculations have been carried out for three frequencies: $10 \mathrm{kHz}, 33.8 \mathrm{MHz}$ and 66 $\mathrm{MHz}$. It can be noted that the inductance of each current loop decreases with the increasing frequency, which is related to the increase of eddy currents in the stator. We can also notice that the inductance value of each current loop are different and depends on the position of the conductor in the slots.

TABLE IV

FE CALCULATION RESULTS OF THE PARAMETER $L_{E S}$

\begin{tabular}{ccccc}
\hline \hline \multirow{2}{*}{ Frequencies } & \multicolumn{5}{c}{ Inductance of the current loops in the "Forward" slot } \\
& \multicolumn{5}{c}{$[\mathrm{nH}]$} \\
\cline { 2 - 5 } & $\mathrm{L}_{\mathrm{es} 1}$ & $\mathrm{~L}_{\mathrm{es} 2}$ & $\mathrm{~L}_{\mathrm{es} 3}$ & $\mathrm{~L}_{\mathrm{es} 4}$ \\
\hline $10 \mathrm{kHz}$ & 301.4 & 248.6 & 196.4 & 68.4 \\
\hline $33.8 \mathrm{MHz}$ & 18.4 & 16.4 & 15.2 & 13.8 \\
\hline $66 \mathrm{MHz}$ & 16.4 & 16.2 & 15.2 & 13.4 \\
\hline
\end{tabular}

At high frequency, the value of each inductance tends to a constant value. This latter is related to the magnetic energy stored in the air gap between the conductor and the slot's walls. Indeed, we can see in Fig. 14, the magnetic field lines remain mainly in the slot.

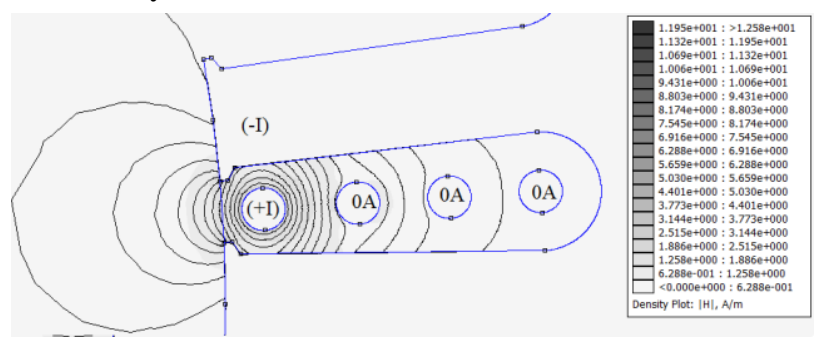

Fig. 14. Equipotential lines and distribution of the magnetic field repartition at $33.8 \mathrm{MHz}$

The inductance parameters $L_{e s}$ ' of the "Return" slot have also been calculated with the same methodology giving the same values because the geometrical positions in the slots are identical.

Strictly speaking, the model should include frequency dependent inductances represented by R-L ladder networks [16]. This feature increases drastically the simulation time. So, for keeping a short simulation time with a reasonable accuracy we decided to keep constant values for the inductances, the ones calculated at $33.8 \mathrm{MHz}$. At this frequency inductive effects become dominant over capacitive ones (see in Fig. 3). The same principle has been retained for the mutual inductance also calculated at $33.8 \mathrm{MHz}$.

Mutual inductance $M_{t \underline{t}}$ : 
The mutual inductance defined hereunder is the one between the current loops identified previously. It is not the mutual inductance between the turns coil, which is not available in a CM configuration.

As an example, the mutual inductance between the current loop (which corresponds to the inductance $\mathrm{L}_{\mathrm{es} 1}$ ), formed by a conductor and the slot's walls, and the current loop (which corresponds to the inductance $\mathrm{L}_{\mathrm{es} 2}$ ), formed by another conductor and the slot's walls, has been calculated as following. Both conductors are set to $1 \mathrm{~A}(+\mathrm{I}=1 \mathrm{~A})$ where all the others conductors are set to zero current. Here the stator is set to $-2 \mathrm{~A}$ current $(-\mathrm{I}=-2 \mathrm{~A})$, because the current that flows through the stator to reach the ground is the sum of all the capacitive currents. After the resolution, the mutual inductance $\mathrm{M}_{\mathrm{te} 1,2}$ is calculated using the expression of the total magnetic energy $\mathrm{W}_{\text {mag } 1,2}(7)$ :

$$
M_{t e 1,2}=\left(W_{m a g 1,2}-\left(\frac{1}{2} L_{e s 1}(+I)^{2}+\frac{1}{2} L_{e s 2}(+I)^{2}\right)\right) /(+I)^{2}
$$

The coupling coefficient is then calculated using equation (8).

$$
K_{1,2}=M_{t e 1,2} / \sqrt{L_{e s 1} L_{e s 2}}
$$

Table $\mathrm{V}$ presents the results of the calculations of the coupling coefficients between one of the current-loop (loop1) and the others in the "forward" slot at $33.8 \mathrm{MHz}$. We can notice that the current loops, where the conductors are adjacent, have the highest coupling coefficient.

TABLE V

CALCULATION RESULTS OF COUPLING COEFFICIENT K IN THE SLOTS

\begin{tabular}{cccccc}
\hline \multirow{2}{*}{ Frequency } & \multicolumn{5}{c}{ Coupling coefficient between the current loops of the } \\
& \multicolumn{5}{c}{ "Forward" slot } \\
\cline { 2 - 6 } & & Loop 1 & Loop 2 & Loop 3 & Loop 4 \\
\hline $33.8 \mathrm{MHz}$ & Loop 1 & $/$ & 0.11 & 0.010 & -0.001 \\
\hline \hline
\end{tabular}

\section{2) Calculation of the inductances in the "Air region"}

In the studied model, each overhang is shaped as a half-loop out of the stator. Thus, we can distinguish two half-loops for each turn of the coil (except for the last turn). Also, these halfloops are identical and all in parallel in each air region.

\section{Inductance $L_{t i}$ :}

The inductance parameter $L_{t t}$ of a half-loop is calculated using analytical formulas[19], the FE method (3D model) and evaluated experimentally. The different results are reported in Table VI.

\section{TABLE VI}

MEASURED VS. FE VS. ANALYTICAL CALCULATION OF $L_{T T}$

\begin{tabular}{cccc}
\hline Inductance $[\mathrm{nH}]$ & Measurement & FE & Analytical \\
\hline $\mathrm{L}_{\mathrm{tt}}$ & 35.2 & 38.0 & 43.0 \\
\hline
\end{tabular}

Experimentally, the inductance parameter $L_{t t}$ is determined from the impedance measurement of an elliptical handcrafted half-loop. This latter has the same geometrical parameter as the half-loops in overhang region of our experimental coil.

Because of analytical formulations [19] and symmetries required for numerical simulation by FE, the half-loop must be considered to be perfectly circular and not elliptical as the experimental shape, with a radius $R_{b}$ of $18.5 \mathrm{~mm}$. However, this radius has been chosen in order to keep the same surfaces between the real device and the equivalent semi-circular loop. This is a reasonable hypothesis in magnetic calculation. This modeling hypothesis explains the differences between the calculation results (Table VI) but they remain acceptable. Inversely to the case of the inductances in iron region, the inductance $L_{t t}$ is constant with frequency.

\section{Mutual inductance $M_{t \underline{t}}$ :}

To calculate the inductive coupling between the half-loops, we used an analytical formula (9) that was presented in [19]. Thereafter, we calculate the coupling coefficient $K_{t t}$ between all the half-loops in the same overhang region. The results are reported in Table VII.

$$
M_{t t}\left(R_{b}, d\right)=\frac{\mu_{0} R_{b}}{L_{t t}} \int_{0}^{\pi} \frac{\left[P_{1} \cos \varphi+P_{2} \cos \varphi+P_{3} \cos \varphi\right] \psi(q)}{q \sqrt{V_{0}^{3}}} d \varphi
$$

Where:

- $d$ is spacing between the half loops

- $P_{1}, P_{2}, P_{3}, q$ and $\mathrm{V}_{0}$ are constants that depended of geometrical parameters

- $\Psi(\mathrm{q})$ is the complete elliptic integral of first kind.

TABLE VII

CALCULATION RESULTS OF COUPLING COEFFICIENT $\mathrm{K}_{\text {TT }}$

\begin{tabular}{ccccc}
\hline \hline \multicolumn{5}{c}{ Coupling coefficient $K_{t t}$} \\
\hline & Half-loop 1 & Half-loop 2 & Half-loop 3 & Half-loop 4 \\
\hline Half-loop 1 & $/$ & 0.55 & 0.38 & 0.28 \\
\hline \hline
\end{tabular}

\section{Calculation of the resistive parameters $R_{s}$ :}

The resistive parameters $R_{s e}$ and $R_{s t}$ are respectively the electric resistance of each conductor in iron region (slots) and in air region (overhang). To take into account the skin and proximity effects which are due to the high frequency working, we used the FE method to calculate these parameters.

As for the inductive parameters, the resistive parameters were only calculated for the critical frequencies $33.8 \mathrm{MHz}$ and $66 \mathrm{MHz}$ (Table VIII).

TABLE VIII

FE CALCULATION RESULTS OF THE RESISTANCE $R_{S E}$ AND $R_{S T}$

\begin{tabular}{ccccc}
\hline \multicolumn{4}{c}{ Resistance parameters $\mathrm{R}_{\mathrm{se}}$ of the conductors in each iron region (slots) $[\Omega]$} \\
\hline Frequency & $\mathrm{R}_{\mathrm{se} 1}$ & $\mathrm{R}_{\mathrm{se} 2}$ & $\mathrm{R}_{\mathrm{se} 3}$ & $\mathrm{R}_{\mathrm{se} 4}$ \\
\hline $33.8 \mathrm{MHz}$ & 0.0129 & 0.0126 & 0.0126 & 0.014 \\
$66 \mathrm{MHz}$ & 0.0178 & 0.0177 & 0.0178 & 0.018 \\
\hline
\end{tabular}

\begin{tabular}{ccccc}
\hline \multicolumn{5}{c}{ Resistance parameters $\mathrm{R}_{\mathrm{st}}$ of the conductors in each air region (overhang) } \\
{$[\Omega]$} & $\mathrm{R}_{\mathrm{st2}}$ & $\mathrm{R}_{\mathrm{st} 3}$ & $\mathrm{R}_{\mathrm{st} 4}$ \\
\hline Frequency & $\mathrm{R}_{\mathrm{st} 1}$ & 0.0079 & 0.0089 & 0.0179 \\
\hline $33.8 \mathrm{MHz}$ & 0.0179 & 0.0116 & 0.0121 & 0.0264 \\
$66 \mathrm{MHz}$ & 0.0264 &
\end{tabular}

As we can see in Table VIII, it is obvious that the resistances increase with frequency. To take into account this frequency dependency, we can also use a voltage dependent current-source circuit element. However, we should calculate the value of the resistance parameters within the frequency band $[10 \mathrm{kHz}$ to $100 \mathrm{MHz}]$ which takes very long $\mathrm{FE}$ simulation time. For this reason, we used a constant value of the resistive parameters that was calculated at $33.8 \mathrm{MHz}$.

\section{DISCUSSION OF THE SIMULATION AND EXPERIMENTAL}

\section{RESULTS}

As was mentioned above, the developed circuit (Fig. 7) is 
simulated with the LTSPICE software. To obtain the frequency response, we used the AC analysis of the software.

Fig. 15 compares the simulated and measured CM impedances of our 4 turns basic experimental coil in a complete 'a priori' simulation based on the previously depicted methodology. Overall, the developed circuit allows predicting the entire impedance spectrum with a good precision at frequencies below $33.8 \mathrm{MHz}$, which confirms the calculated value of the stray capacitances. Therefore, the shift between the critical frequencies (resonance and antiresonance) of the two curves is related to some uncertainties in estimation of the inductive parameters (values of inductance and mutual), which are introduced by the simplifying hypothesis used (constant inductance value in iron region, considering perfect half loops in air region, etc.).

However, we can notice that the damping coefficients of the simulated curve, at critical frequencies, differ from those measured. This mismatch can be explained as follows.

The damping is governed by losses; resistive losses, dielectric losses and Iron losses. Regarding the resistive losses (parameters $R_{s}$ ), the FE method used on calculation of these parameters gives a good estimation. Nevertheless, the frequency dependence of these parameters has not been taken into account in our simulation, where only the value at the resonance frequency was used.
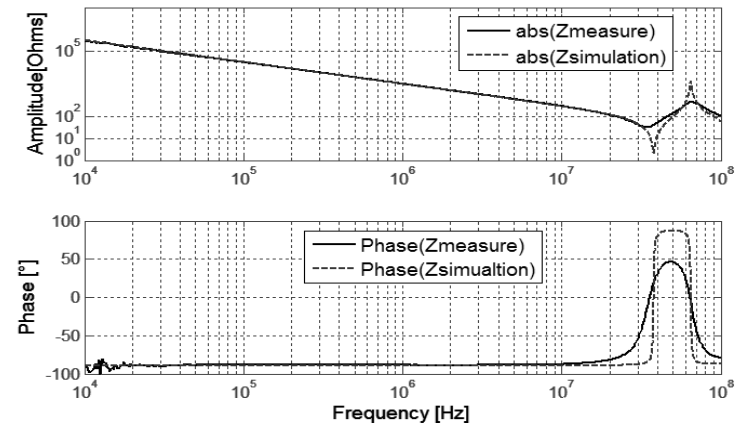

Fig. 15. Measured vs. predicted CM impedance of the experimental coil

Regarding the dielectric losses (parameters $R_{p}$ ), the frequency dependence has been taken into account. However, because of the difficulty to estimate these parameters at the first stage of modeling, the equivalent value used is not accurately representative of the dielectric losses. Furthermore, the iron losses have not been considered.

In order to improve the model prediction, we have done a new identification of parameter $R_{p}$ from the measured curve thanks to an optimization software. The new numerical function of $R_{p}$ has the following expression (10):

$$
R_{p}(f)=T F_{p}(s)=4 * 10^{15} *\left(\frac{s}{j * 2 * \pi}\right)^{-1.63}
$$

As we can observe in Fig. 16, the new prediction of the CM impedance is now in good agreement with the measurement both in low- and high- frequency ranges. We can also note that the damping is acting naturally on the critical frequencies.
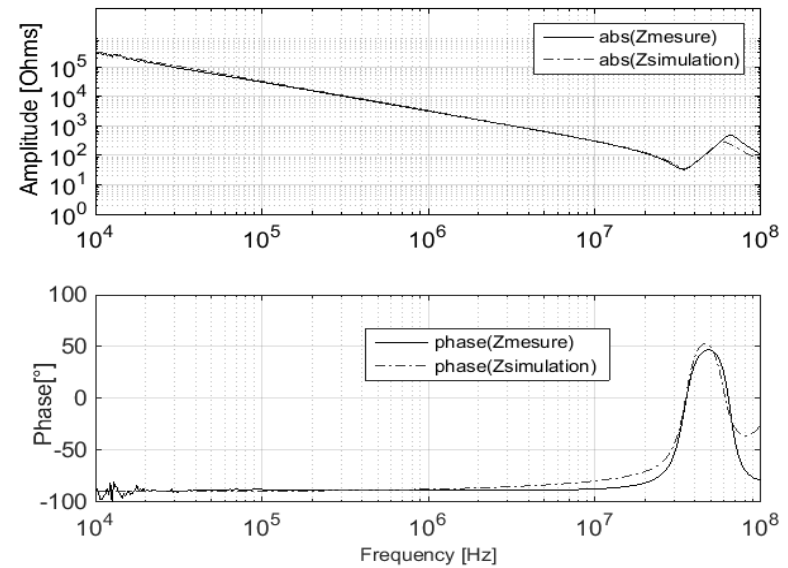

Fig. 16. Measured vs. predicted CM impedance of the experimental coil, with correction on the dielectric losses model

Now that we have validated the proposed methodology for one coil, let's check it for the case of two identical coils. These latter are placed in the stator, with a gap of two teeth, and connected in series. In this case, the electrical model of this configuration consists of the series arrangement of the electrical circuit of each coil, which has been established in the previous step. Also, the value of all parameters determined previously was retained.

It has to be noted that the two coils in this disposition are weakly coupled especially at high frequency; because the field lines are confined in the slots. For this reason, and also because the capacitive couplings are dominant at low frequency, the inductive couplings between these coils are neglected in the modeling.

The prediction results are compared to measurements in Fig. 17. We can see that the proposed model predicts correctly the CM impedance of the two series coils with good accuracy over all the frequency range.

In the same way, we established the electrical model of the 7 coils presented in Fig. 1(b) wounded on the entire stator slots. This model consists of the series arrangement of 7 electrical basic circuits.
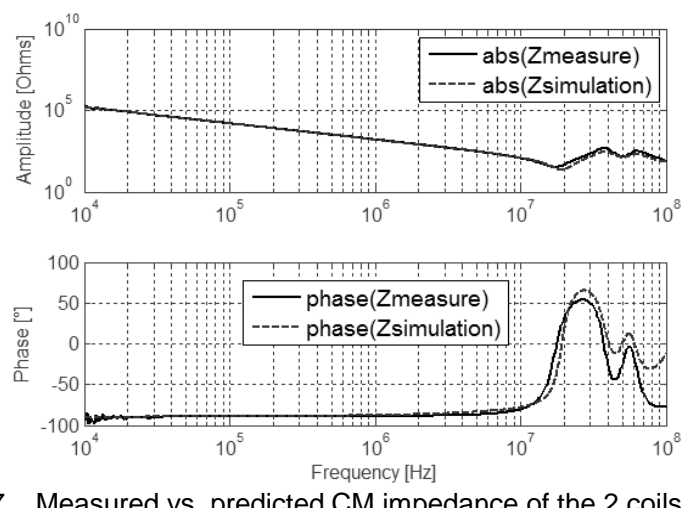

Fig. 17. Measured vs. predicted CM impedance of the 2 coils

As we can observe in Fig. 18, the proposed model allows predicting the $\mathrm{CM}$ impedance with a satisfying agreement with the measurement. However, as would be expected, we can notice that the critical frequencies of the simulation curve are shifted right compared to those of the measured curve. Indeed, the values used for the inductive parameters in iron 
regions was the ones calculated at $33.8 \mathrm{MHz}$, while the first resonance of the impedance of the seven coils is $4.8 \mathrm{MHz}$. So, the real inductances are greater. For this purpose, we have carried out a new simulation where the inductive parameters are calculated at $4.8 \mathrm{MHz}$. The simulation results are reported in Fig. 19. As we can see, the first resonance frequency at 4.8 $\mathrm{MHz}$ is now well predicted. However, the higher resonances frequencies are now a little bit shifted. This feature highlights the need of nonlinear modeling of inductances. Despite this shift, the prediction of the CM impedance up to $100 \mathrm{MHz}$ remains satisfactory, validating our methodology.

In view of all these investigations, the improvement of these results could be possible by introducing a non-linear model of both the inductance element and mutual inductance. These latter are not available presently and will be the subject of further works.
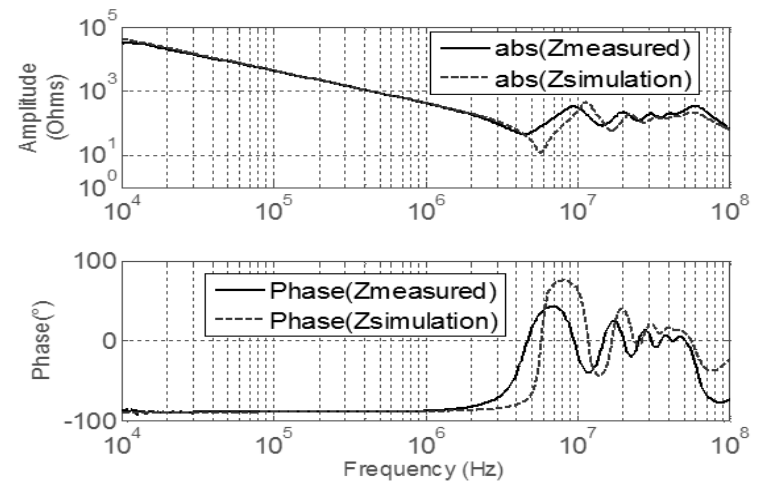

Fig. 18. Measured vs. predicted $\mathrm{CM}$ impedance of 7 coils, with inductance parameters calculated at $33.8 \mathrm{MHz}$
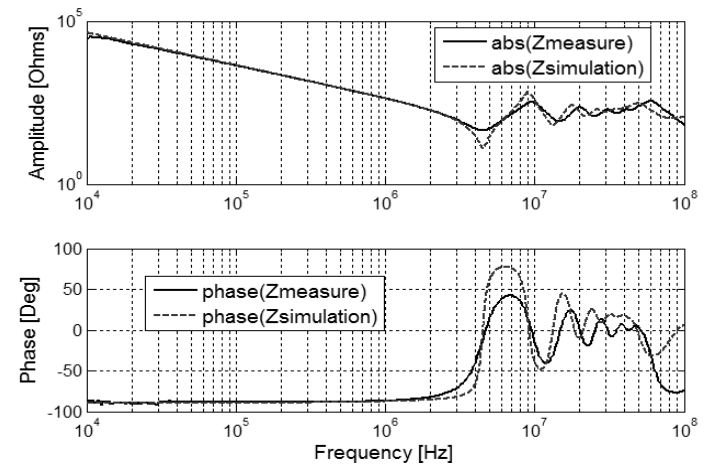

Fig. 19. Measured vs. predicted $\mathrm{CM}$ impedance of 7 coils, with inductance parameters calculated at $4.8 \mathrm{MHz}$

\section{CONCLUSION}

In this paper we presented a strategy for predictive modeling of the CM impedance of the stator coils in AC machines. However, this proposed strategy can also be used for differential-mode analysis.

The original aspects of this work are, on the one hand, the separation of electromagnetic phenomena in the stator from those in overhangs region, what allows to apprehend their disparities, and then to model each region by a lumped parameters circuit with physical meaning. This requires that the length of the modeled parts is much smaller than the wavelength at the highest frequency of the studied phenomena. On the other hand, our methodology has proposed to take into account the frequency dependence of the dielectric losses and to identify accurately all the inductive phenomena, frequency dependant, involved in the CM impedance.

The developed model, based on this methodology, has allowed having a satisfactory prediction of the CM impedance of the experimental winding, which is composed of simplified coils, within the frequency band $[10 \mathrm{kHz}$ to $100 \mathrm{MHz}]$; the low-frequency capacitance is predicted with better than $5 \%$ accuracy, while the peaks and dips in the CM impedance plot are very well predicted beyond $5 \mathrm{MHz}$.

In view of these results, it would be perfectly possible to use this methodology for global modeling: common and differential mode. On the other hand, combining the generated "netlist" with optimization routines, it would be also possible to control the CM impedance at the first stage of the motor conception.

Furthermore, this work has highlighted the need of the characterization of the dielectrics and magnetic materials at high frequency in order to have a good estimation of all losses and therefore a good prediction of the impedance damping.

\section{ACKNOWLEDGMENTS}

The authors thank all partners involved in the program VTT E-CEM. This work has been supported by the National Agency for Research, program VTT E-CEM, ref: ANR-10VPTT-0013.

\section{REFERENCES}

[1] F. Costa, C. Vollaire, and R. Meuret, "Modeling of Conducted Common Mode Perturbations in Variable-Speed Drive Systems," IEEE Trans. Electromagn. Compat., vol. 47, no. 4, pp. 1012-1021, Nov. 2005.

[2] J. Erdman, R. J. Kerkman, D. Schlegel, and G. Skibinski, "Effect of PWM inverters on AC motor bearing currents and shaft voltages,", in Applied Power Electronics Conference and Exposition, 1995. APEC '95. Conference Proceedings 1995., Tenth Annual, vol. 1, pp. 24-33, 1995.

[3] D. F. Busse, J. M. Erdman, R. J. Kerkman, D. W. Schlegel, and G. L. Skibinski, "The effects of PWM voltage source inverters on the mechanical performance of rolling bearings," IEEE Trans. Ind. Appl., vol. 33, no. 2, pp. 567-576, Mar/Apr 1997.

[4] G. Carlo M. Okubo, Hitoshi, Naoki Hayakawa, "technical development on partial discharge measurement and electrical insulation techniques for low voltage motors driven by voltage inverters," IEEE Trans. Dielectr. Electr. Insul., vol. 14, no. 6, pp. 1516 - 1530, Dec. 2007.

[5] M. Kaufhold and H. Aninger, "Electrical stress and failure mechanism of the winding insulation in PWM-inverter-fed low-voltage induction motors," IEEE Trans. Ind. Electron., vol. 47, no. 2, pp. 396-402, Apr. 2000.

[6] U. Shami, "Identification and discussion of the origin of a shaft end-toend voltage in an inverter-driven motor," IEEE Trans. Power Electron., vol. 25 , no. 6, pp. 1615-1625, Jun. 2010.

[7] S. Chen and T. A. Lipo, "Circulating type motor bearing current in inverter drives,", in conference Record of the 1996 IEEE Industry Applications Conference Thirty-First IAS Annual, vol. 1, pp. 162-167, Oct. 1996.

[8] A. Muetze, A. Binder, H. Vogel, and J. Hering, "Experimental evaluation of the endangerment of ball bearings due to inverter-induced bearing currents," , in Industry Applications Conference, 2004. 39th IAS Annual Meeting. Conference Record of the 2004 IEEE, vol. 3, no. 1, pp. 19891995. Oct. 2004

[9] A. Boglietti, A. Cavagnino, and M. Lazzari, "Experimental HighFrequency Parameter Identification of AC Electrical Motors," IEEE Trans. Ind. Appl., vol. 43, no. 1, pp. 23-29, Jan.-feb. 2007.

[10]M. Degano, P. Zanchetta, L. Empringham, E. Lavopa, and J. Clare, "HF induction motor modeling using automated experimental impedance measurement matching," IEEE Trans. Ind. Electron., vol. 59, no. 10, pp. 3789-3796, Oct. 2012. 
[11]R. Thottappillil, M. Enohnyaket, K. Jia, and G. Bohlin, "Modelling an AC motor with high accuracy in a wide frequency range," IEEE, IET Electr. Power Appl., vol. 7, no. 2, pp. 116-122, Feb. 2013.

[12]G. Vidmar and D. Miljavec, "A universal high-frequency three-phase electric-motor model suitable for the delta and star winding connections," IEEE Trans. Power Electron., vol. 8993, no. c, pp. 1-1, août 2014.

[13]G. Suresh and H. Toliyat, "Predicting the transient effects of PWM voltage waveform on the stator windings of random wound induction motors," IEEE Trans. POWER Electron., vol. 14, no. 1, pp. 23-30, Jan. 1999.

[14]K. Maki, H. Funato, and L. Shao, "Motor modeling for EMC simulation by 3-D electromagnetic field analysis,", in IEEE International Electric Machines and Drives Conference, 2009. IEMDC '09., pp. 103-108, May 2009.

[15] V. Mihaila, S. Duchesne, and D. Roger, "A simulation method to predict the turn-to-turn voltage spikes in a PWM fed motor winding," IEEE Trans. Dielectr. Electr. Insul., vol. 18, no. 5, pp. 1609-1615, Oct. 2011.

[16]F. Abdallah and M. Alakula, "Inductive analysis of a multiconductor system prototype,"IEEE, in 2014 International Symposium on Electromagnetic Compatibility, pp. 1170-1175, Sept. 2014.

[17] A. Muetze and A. Binder, "Calculation of Circulating Bearing Currents in Machines of Inverter-Based Drive Systems," IEEE Trans. Ind. Electron., vol. 54, no. 2, pp. 932-938, Apr. 2007.

[18]FEMM software website, "http://www.femm.info/wiki/HomePage" .

[19]F. W. GROVER, "Inductance calculations: Working Formulas and Tables", New York: Dover, Jun. 1982.

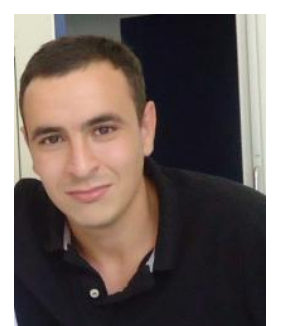

Nidhal Boucenna (S'12) was born in Algeria, in 1988. He received the M.S. degrees in electrical engineering from the University of Claude Bernard and Ecole Centrale de Lyon France in 2010. He also received the Ph.D. degree in electrical engineering from the Ecole Normale Superieure de Cachan (Ens cachan FRA) in 2014.

His main research interest are electromagnetic modeling and EMC analysis of electrical motors (automotive and airplane applications).

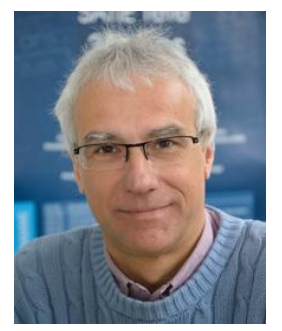

François Costa (M'95) received the Ph.D. in electrical engineering from the University of Paris-Sud, Orsay, France, in 1992. He is a Full Professor in University Paris Est Créteil, since 2003, where he is responsible for the master degree in education (sciences \& technology) in the "Ecole Supérieure de l'Education et du Professorat (ESPE)". Since 2013, he is heading the department "components \& systems for electrical energy" (CSEE) of lab SATIE (35 people, 40 PhD students). His research fields include the high-frequency medium-power converters, the EMI issues and modelling, the integration in power electronics, the piezoelectric converters and low-level energy harvesting systems. His main activities are focused on EMI in power converters and systems: multiscale modelling of conducted and radiated emissions, mitigation techniques, EMI modelling of actuators. He is the author or co-author of more than 60 journal papers, 3 books and more than 180 conference papers.

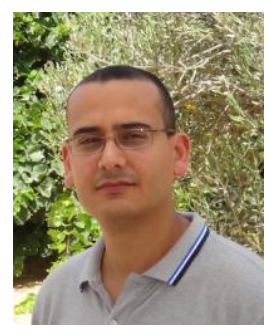

Sami Hlioui (M'08) is lecturer in the Conservatoire National des Arts et Métiers in Paris (Le CNAM - Paris - France) and researcher in the SATIE laboratory of the Ecole Normale Supérieure de Cachan - France (ENS de Cachan). He obtained his electromechanical engineer diploma from the National School of Engineering of Sfax - Tunisia (ENIS) in 2004, his M.Sc. degree in electrical engineering from the ENS de Cachan - France and his Ph.D. degree in electrical power engineering from the University of Technology of Belfort-Montbeliard - France (UTBM) in 2008.

His main research interest are the multidisciplinary modeling of electromagnetic actuators (electromagnetic modeling in low $(0 . .10 \mathrm{kHz})$ and high $(0 . .2 \mathrm{MHz})$ frequency domain, thermal and mechanical models) and the optimal design of these actuators for embedded applications (automotive and airplane applications).

He currently participates in several research programs and supervises different $\mathrm{PhD}$ thesis.

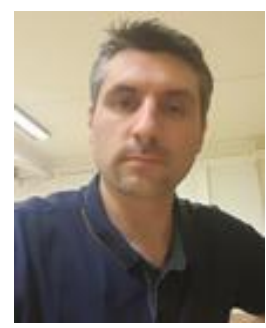

Bertrand Revol (M'12) was born on June 11th, 1976 in France. He obtained his engineer degree of ENSIEG from the INPG (Institut National Polytechnique de Grenoble) in June 2000. Since November 2003 he is a holder of an electrical engineering Ph.D. from the Joseph Fourier University dealing with the Electromagnetic Compatibility (EMC) in power electronics. These principal fields of research relate to the EMC of the power electronics converters and to the modelling of structures and components. Since September 2004, he has been an Assistant Professor at the Ecole Normale Supérieur de Cachan (ENSC), Cachan, France, where he carries out research in the laboratory of SATIE and works on the improvement of EMI issues and modelling for optimization and integration in power electronics. 